\title{
ESTUDO NUMÉRICO E ANALÍTICO DE UM ENSAIO DE FRATURAMENTO HIDRÁULICO
}

\section{NUMERICAL AND ANALYTICAL STUDY OF A HYDRAULIC FRACTURE TEST}

\author{
G. SIMONELLI ${ }^{1, *}$, L. S. SILVA ${ }^{1}$, R. S. VILARES FILHO ${ }^{1}$ e P. G. C. LINS ${ }^{1}$
}

${ }^{1}$ Universidade Federal da Bahia, Departamento de Ciência e Tecnologia dos Materiais, Salvador, Bahia, Brasil

${ }^{*}$ Autor para correspondência: Universidade Federal da Bahia, Departamento de Ciência e Tecnologia dos Materiais, Salvador, Bahia, Brasil, Phone: +5571 32839854

Endereço de e-mail: gsimonelli@ufba.br

\begin{tabular}{|c|}
\hline A R T I C LE I N F O \\
\hline $\begin{array}{l}\text { Article history: } \\
\text { Received 2018-10-11 } \\
\text { Accepted 2018-12-20 } \\
\text { Available online 2019-04-25 }\end{array}$ \\
\hline $\begin{array}{l}\text { palavras-chave } \\
\text { Fraturamento hidráulico } \\
\text { Elasticidade } \\
\text { Elementos finitos } \\
\text { Mecânica da fratura }\end{array}$ \\
\hline $\begin{array}{l}\text { keywords} \\
\text { Hydraulic fracturing } \\
\text { Elasticity } \\
\text { Finite elements } \\
\text { Fracture mechanics }\end{array}$ \\
\hline
\end{tabular}

RESUMO

Os resultados de um ensaio de fraturamento hidráulico de laboratório são analisados por modelos da elasticidade e da mecânica da fratura. Uma solução analítica para uma abertura circular em meio infinito com tensões in situ não hidrostáticas, uma carga interna e um modelo de elementos finitos do ensaio apresentam tensões de tração máximas que superam a resistência à tração do material. Estes dois modelos explicam a iniciação da fratura. Soluções de referência de mecânica da fratura e modelos de elementos finitos para representar o fraturamento no ensaio, quando comparados com tenacidade à fratura, explicam o processo de propagação da fratura. O conjunto dos modelos permite um melhor entendimento do processo da mecânica do fraturamento. As tensões in situ, a pressão interna do furo e a pressão na fratura possuem papeis distintos no referido processo.

\footnotetext{
ABSTRACT

The results of a laboratory hydraulic fracturing test are analyzed by models of elasticity and fracture mechanics. An analytical solution for an infinite medium circular aperture with nonhydrostatic in situ stresses, an internal load and a finite element model of the test exhibits maximum tensile stresses that exceed the tensile strength of the material. These two models explain the initiation of the fracture. Fracture mechanics reference solutions and finite element models to represent fracture in the test, when compared to fracture toughness, explain the fracture propagation process. The set of models allows a better understanding of the process of fracturing mechanics. The stresses in situ, the internal pressure of the hole and the pressure in the fracture have different roles in said process.
} 


\section{INTRODUÇÃO}

O fraturamento hidráulico é uma operação realizada na indústria de petróleo que permite aumentar a produção de reservatórios de baixa permeabilidade (ZHAO et al., 2018). O fraturamento hidráulico pode ser utilizado também para medir as tensões in situ (geopressões).

O procedimento de fraturamento hidráulico pode ser interpretado com a hipótese de fluido não penetrante ou de fluido penetrante. Cada uma destas hipóteses é mais realística dependendo das características de permeabilidade da rocha do entorno do poço. No presente trabalho, um ensaio de laboratório de fraturamento hidráulico realizado em um mármore é interpretado com teorias da mecânica do contínuo e da mecânica da fratura. As características do mármore fazem plausível a análise com a hipótese de fluido não penetrante.

Nas análises considerando o meio contínuo as tensões atuantes são comparadas com um critério de resistência do material para verificar a ruptura ou fraturamento do material. A mecânica da fratura considera que a ruptura do material se dá pela propagação de fraturas pré-existentes.

O objetivo geral do trabalho é realizar uma intepretação mecanicista de um ensaio de fraturamento hidráulico de laboratório. Como objetivos específicos se tem: (a) analisar a iniciação da fratura por um modelo analítico e um modelo de elementos finitos; e (b) analisar o processo de propagação da fratura com um modelo de referência e um modelo de elementos finitos.

\section{METODOLOGIA}

Uma série de experimentos de fraturamento hidráulico em laboratório foi realizada por Haimson (1968) e discutidos em Haimson e Fairhurst (1970). Uma parte dos experimentos foi realizada em cubos de 5 polegadas $(0,127 \mathrm{~m})$ de aresta e um furo de 0,3 polegadas $(0,00762 \mathrm{~m})$ de diâmetro. Cada cubo era colocado em um pórtico com quatro macacos planos nas laterais para simular as tensões horizontais in situ e colocado em uma prensa para simular a tensão vertical in situ. A Figura 1(a) mostra o pórtico de ensaio. Obturadores eram colocados na extremidade do furo para permitir a aplicação de uma pressão interna por meio de um fluido (óleo lubrificante).

No presente trabalho tomou-se como referência o ensaio realizado na amostra número 24 . A rocha desta amostra era um mármore Tennesse. As tensões aplicadas no plano horizontal foram $\sigma_{\mathrm{x}}{ }^{\mathrm{o}}=1300 \mathrm{psi}(8,963 \mathrm{MPa}) \sigma_{\mathrm{y}}{ }^{\mathrm{o}}=2000 \mathrm{psi}(13,790 \mathrm{MPa})$; na vertical $\sigma_{\mathrm{z}}{ }^{\circ}=1650 \mathrm{psi}(11,376 \mathrm{MPa})$. A amostra fraturou na vertical com uma pressão no furo $\mathrm{p}_{\mathrm{w}}=4450 \mathrm{psi}(30,682 \mathrm{MPa})$, em um padrão que pode ser observado na Figura 1(b).

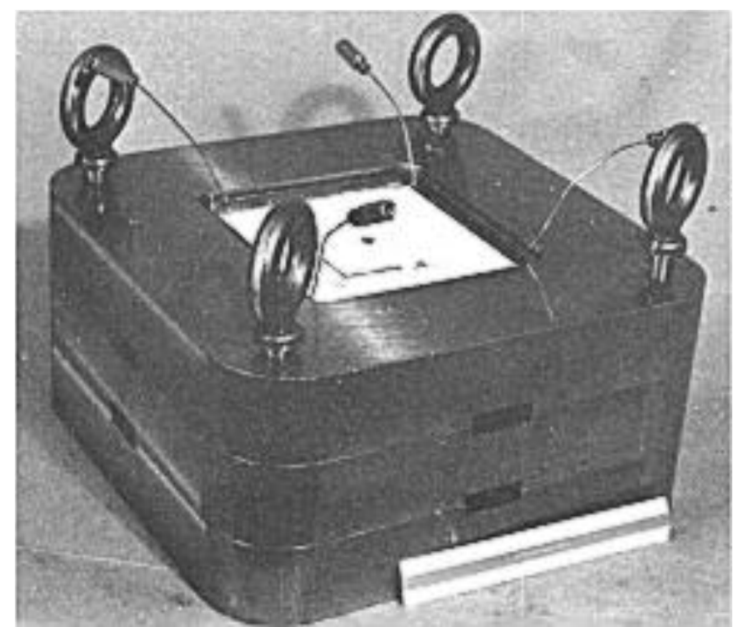

(a)

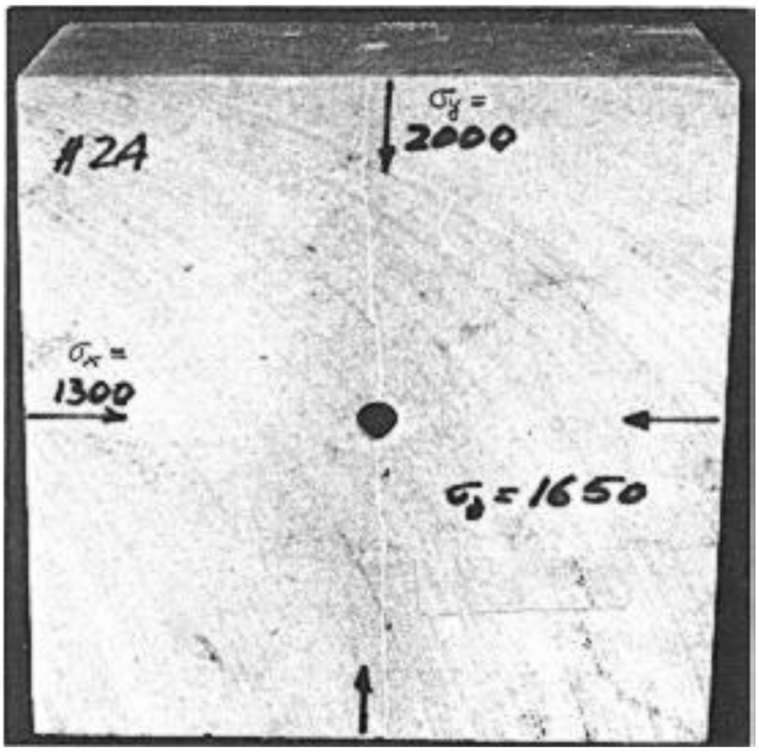

(b)

Figure 1 - (a) Pórtico de aço com amostra de rocha e quatro macacos planos; (b) Seção horizontal mostrando fratura vertical em amostra de mármore Tennesse (HAIMSON \& FAIRHURST, 1970).

O trabalho de Bradley (1979) é uma referência padrão para a distribuição de tensões ao redor de um poço inclinado, escavado em um maciço elástico linear. $\mathrm{O}$ desenvolvimento desta classe de soluções é discutido em Fjaer et al. (2008), cuja notação é adotada no presente trabalho. As tensões in situ são dadas por $\left\{\sigma_{\mathrm{x}}{ }^{\mathrm{o}},{\sigma_{\mathrm{y}}}^{\mathrm{o}},{\sigma_{\mathrm{z}}}^{\mathrm{o}},{\sigma_{\mathrm{xy}}}^{\mathrm{o}},{\sigma_{\mathrm{xz}}}^{\mathrm{o}},{\sigma_{\mathrm{yz}}}^{\mathrm{o}}\right\}$. O poço possui um raio $\mathrm{R}_{\mathrm{w}}$. Na parede do poço uma pressão interna $\mathrm{p}_{\mathrm{w}}$ é aplicada. A solução é dada em termos de um sistema de coordenadas polares definida por um raio $r$ e um ângulo $\theta$, dado em sentido antihorário com relação ao eixo de coordenadas x. A solução é constituída pelas equações a seguir: 
$\sigma_{r}=\frac{\sigma_{x}^{o}+\sigma_{y}^{o}}{2}\left(1-\frac{R_{w}^{2}}{r^{2}}\right)+\frac{\sigma_{x}^{o}-\sigma_{y}^{o}}{2}\left(1+3 \frac{R_{w}^{4}}{r^{4}}-4 \frac{R_{w}^{2}}{r^{2}}\right) \cos 2 \theta+\tau_{x y}^{o}\left(1+3 \frac{R_{w}^{4}}{r^{4}}-4 \frac{R_{w}^{2}}{r^{2}}\right) \sin 2 \theta+p_{w} \frac{R_{w}^{2}}{r^{2}}$

$\sigma_{\theta}=\frac{\sigma_{x}^{o}+\sigma_{y}^{o}}{2}\left(1+\frac{R_{w}^{2}}{r^{2}}\right)-\frac{\sigma_{x}^{o}-\sigma_{y}^{o}}{2}\left(1+3 \frac{R_{w}^{4}}{r^{4}}\right) \cos 2 \theta-\tau_{x y}^{o}\left(1+3 \frac{R_{w}^{4}}{r^{4}}\right) \sin 2 \theta-p_{w} \frac{R_{w}^{2}}{r^{2}}$

$\sigma_{z}=\sigma_{z}^{o}-v_{f r}\left[2\left(\sigma_{x}^{o}-\sigma_{y}^{o}\right) \frac{R_{w}^{2}}{r^{2}} \cos 2 \theta+4 \tau_{x y}^{o} \frac{R_{w}^{2}}{r^{2}} \sin 2 \theta\right]$

$\tau_{r \theta}=\frac{\sigma_{y}^{o}-\sigma_{x}^{o}}{2}\left(1-3 \frac{R_{w}^{4}}{r^{4}}+2 \frac{R_{w}^{2}}{r^{2}}\right) \sin 2 \theta+\tau_{x y}^{o}\left(1-3 \frac{R_{w}^{4}}{r^{4}}+2 \frac{R_{w}^{2}}{r^{2}}\right) \cos 2 \theta$

$\tau_{\theta z}=\left(-\tau_{x z}^{o} \sin \theta+\tau_{y z}^{o} \cos \theta\right)\left(1+\frac{R_{w}^{2}}{r^{2}}\right)$

$\tau_{r z}=\left(\tau_{x z}^{o} \cos \theta+\tau_{y z}^{o} \sin \theta\right)\left(1-\frac{R_{w}^{2}}{r^{2}}\right)$

Um simulador que permite calcular os termos da solução de BRADLEY (1979) foi implementado no Ambiente Integrado de Desenvolvimento Lazarus (LAZARUS AND FREE PASCAL TEAM, 2014). O simulador calcula a solução analítica em uma malha que é exportada para o pré e pósprocessador genérico de elementos finitos GiD (CIMNE, 2017).

Um modelo de elementos finitos para o ensaio de laboratório sem a fratura foi elaborado no programa FRANC2D (WAWRZYNEK \& INGRAFFEA, 1993). O lado esquerdo do modelo teve o deslocamento horizontal impedido, e o lado inferior do modelo teve o deslocamento vertical impedido. No lado superior foi aplicada uma carga $\sigma_{\mathrm{x}}{ }^{\circ}=8,963 \mathrm{MPa}$, no lado direito foi aplicada uma carga $\sigma_{\mathrm{y}}{ }^{\mathrm{o}}=13,790 \mathrm{MPa}$, e no furo interno foi aplicada uma pressão $\mathrm{p}_{\mathrm{w}}=30,682 \mathrm{MPa}$.

Em uma abordagem da mecânica da fratura o esforço na ponta da fratura pode ser representado por um fator de intensidade de tensão. A fratura se propaga se o fator de intensidade de tensão for maior que a tenacidade à fratura do material. A propagação da fratura pode ser por tração (modo I), cisalhamento (modo II) e rasgamento (modo III). O presente trabalho focou o estudo na propagação por tração (modo I).

Uma série de simulações representando o crescimento da fratura foi realizada no programa FRANC2D. Os comprimentos de fratura simulados foram de 0,$69 ; 4,19 ; 6,19$; 16,19; 26,19 e 36,19 milímetros. Os resultados das simulações no FRANC2D em termos de fatores de intensidade de tensão foram comparados com uma solução de referência apresentada em Tada et al., (2000), representada na Figura 2.

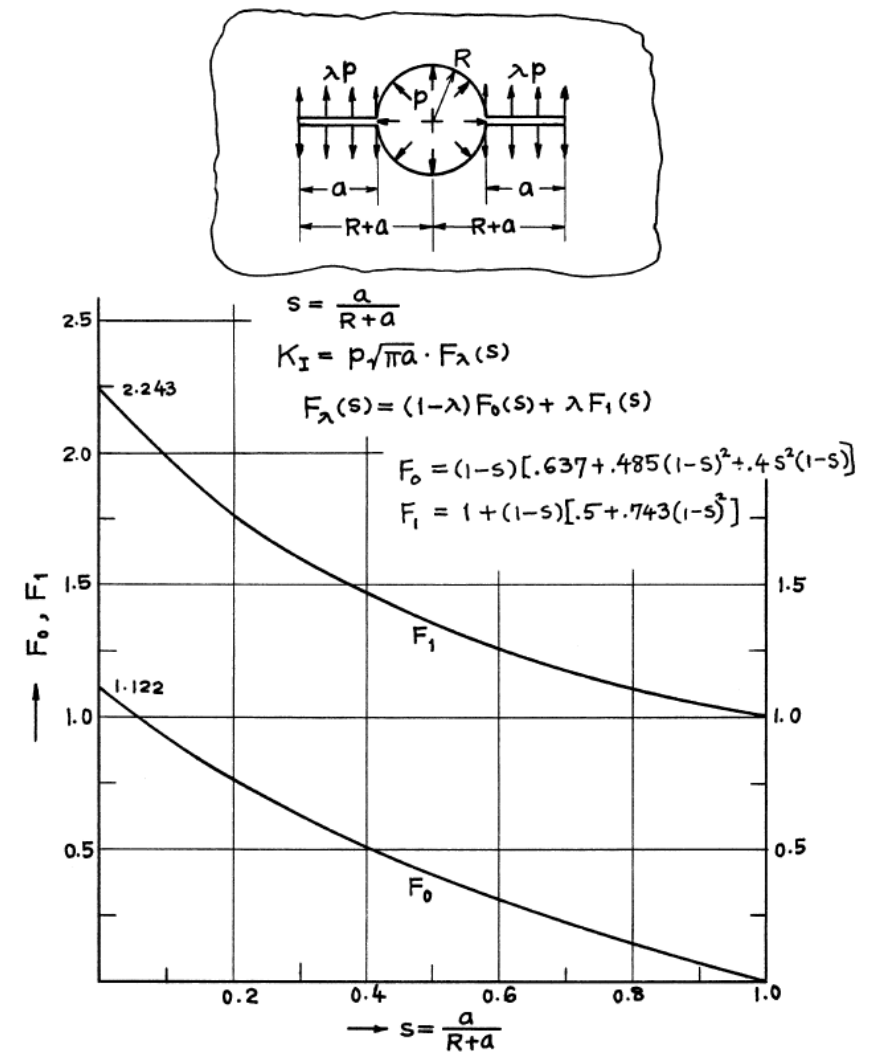

Figure 2 - Solução de referência derivada numericamente (TADA et al., 2000). 


\section{RESULTADOS E DISCUSSÃO}

A Figura 3 apresenta a solução de Bradley (1979) referidos ao sistema de coordenadas cartesianas para os esforços aplicados no ensaio da amostra 24 de Haimson (1968). Os resultados da simulação por elementos finitos com o programa FRANC2D referidos ao sistema de coordenadas cartesianas são apresentados na Figura 4. Estes resultados numéricos podem ser comparados com os da Solução de Bradley (1979), constante na Figura 3. Deve ser destacado que a solução de elementos finitos se refere a um modelo de dimensões do corpo de prova, e a solução de Bradley (1979) refere-se a uma abertura circular em um meio infinito. A comparação entre as Figuras 3 e 4 indicam distribuições de tensões similares.

As soluções sem fratura permitem definir um critério de iniciação da fratura, comparando a tensão de tração nos modelos com a resistência a tração do material. A Tabela 1 apresenta os parâmetros físicos do mármore Tennesse, conforme Haimson (1968). A tensão de tração máxima nos modelos contínuos se situa na parede do poço $\left(r=\mathrm{R}_{\mathrm{w}}\right)$, em $\theta=90^{\circ}$ e $\theta=270^{\circ}$. Pode-se observar na Figura 3(a) a tensão de tração máxima para a solução de Bradley (1979) é de 17,583 MPa. Pode-se observar na Figura 4(a) que na solução de elementos finitos do programa FRANC2D é de 16,04 MPa. Quando se compara estes valores com a resistência a tração obtida pelo ensaio brasileiro a iniciação da fratura é justificada.

As correlações apresentadas por Backers (2004) foram utilizadas para estimar a tenacidade à fratura modo I para o mármore Tennesse, conforme Tabela 2. Na modelagem no FRANC2D (Figura 5) o carregamento foi dividido em tensões externas (LC1), pressão no furo (LC2) e pressão na fratura (LC3). A Tabela 3 apresenta os fatores de intensidade de tensão para cada parcela de carregamento. Os fatores de intensidade de tensão de $\mathrm{LC} 1$ foram negativos, isto é, a fratura fecha devido a este carregamento. Uma análise colocando um elemento finito de interface na fratura poderia melhorar o modelo.

O estudo de Sammis e Ashby (1986) contempla o crescimento de uma fratura a partir de um furo circular em um meio infinito com um carregamento não hidrostático, mas comparações com estes modelos não foram consistentes. Sammis e Ashby (1986) destacam que só ocorre tração devido as tensões externas quando a relação entre $\sigma_{\mathrm{x}}{ }^{\circ} / \sigma_{\mathrm{y}}{ }^{0}$ é inferior a $1 / 3$; no presente caso esta relação é de 0,65 . A combinação $\mathrm{LC} 2+\mathrm{LC} 3$ apresenta valores coerentes de fatores de intensidade de tensão com os valores obtidos na solução de referência de Tada et al., (2000). As tenacidades à fratura estimadas pelas correlações de Backers (2004) indicam valores entre 2,48 e 3,59. A propagação da fratura pode ser justificada pelo limite inferior destes valores.

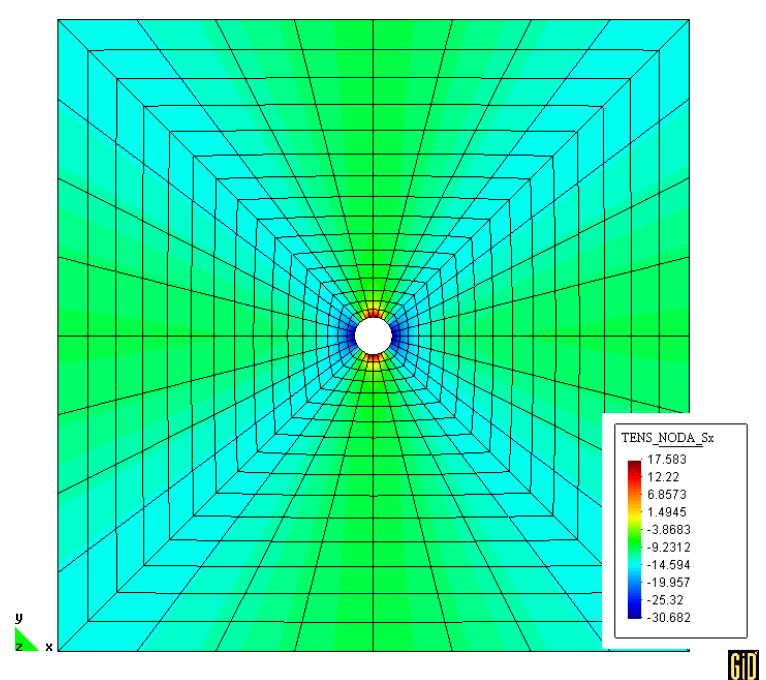

(a) $\sigma_{\mathrm{x}}$

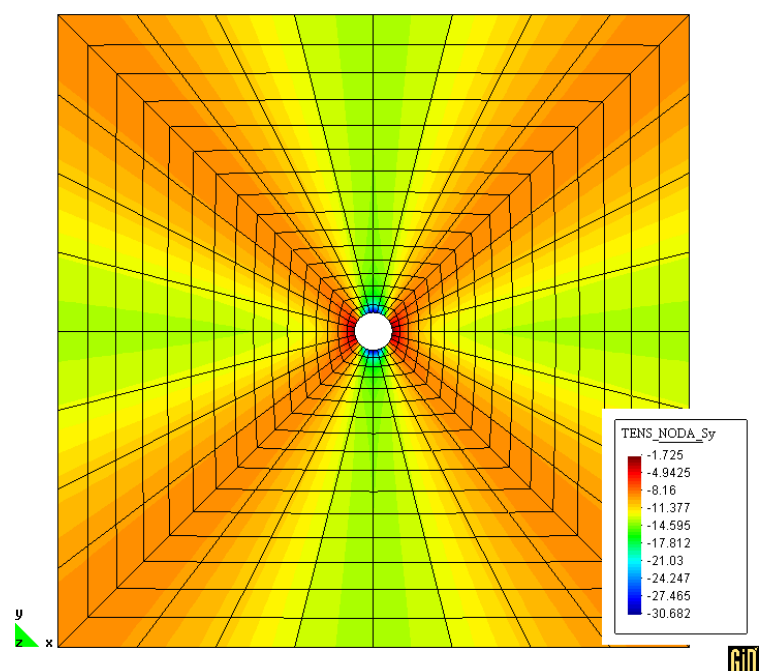

(b) $\sigma_{y}$

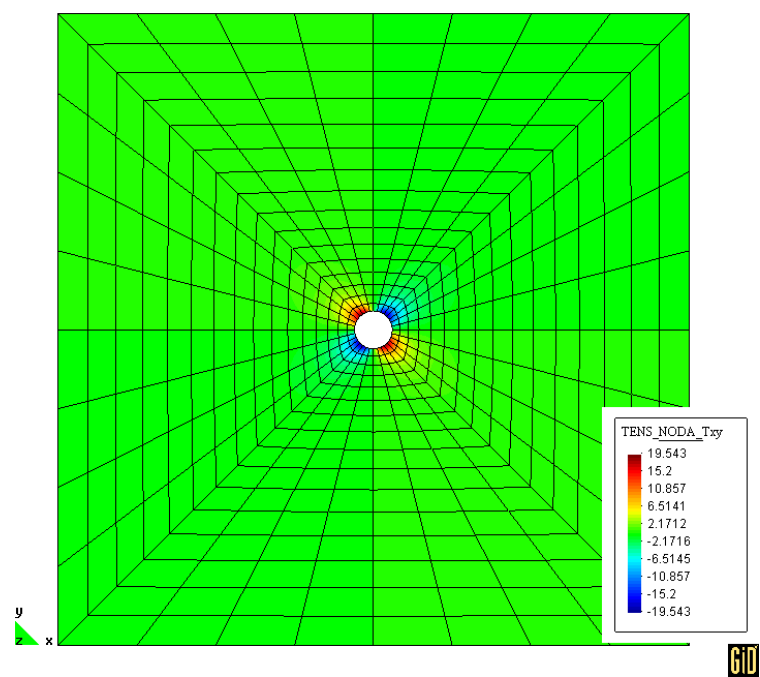

(c) $\tau_{\mathrm{xy}}$

Figure 3 - Solução de Bradley (1979) referida ao sistema de coordenadas cartesianas. 


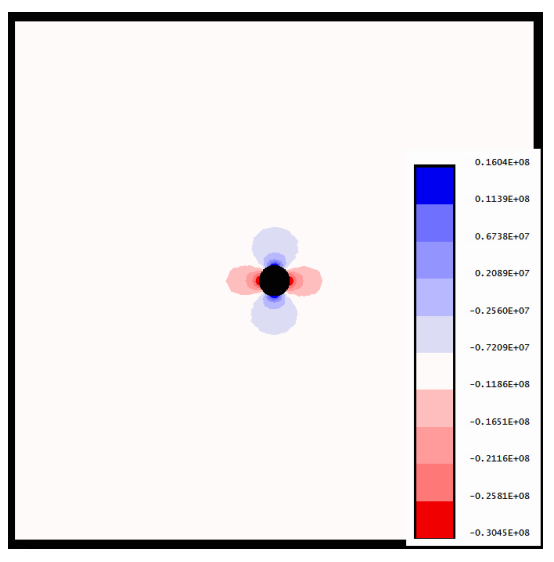

(a) $\sigma_{\mathrm{x}}$

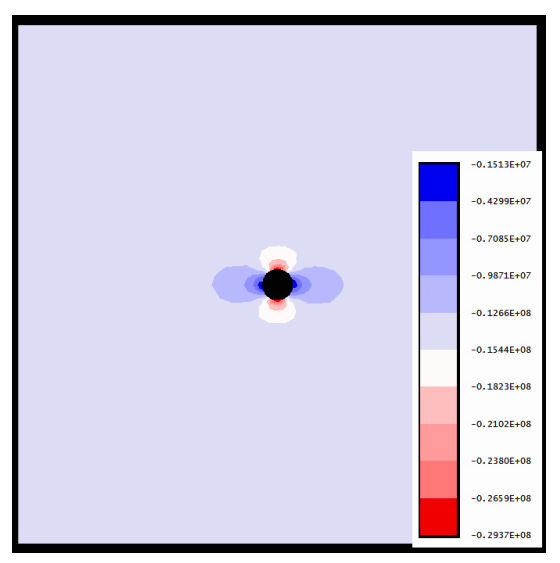

(b) $\sigma_{\mathrm{y}}$

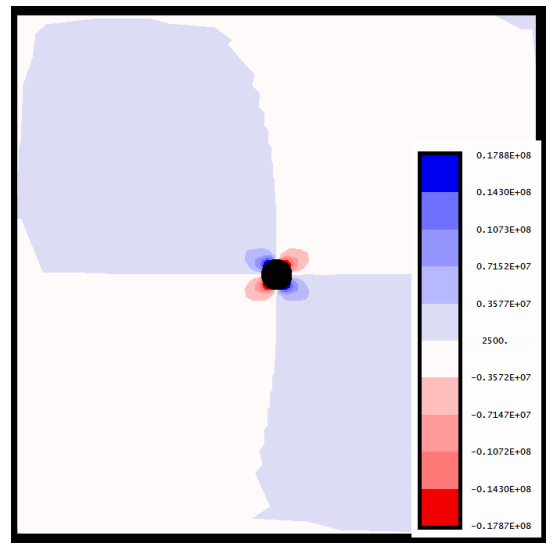

(c) $\tau_{\mathrm{xy}}$

Figure 4 - Solução numérica sem fratura do programa FRANC2D referida ao sistema de coordenadas cartesianas.

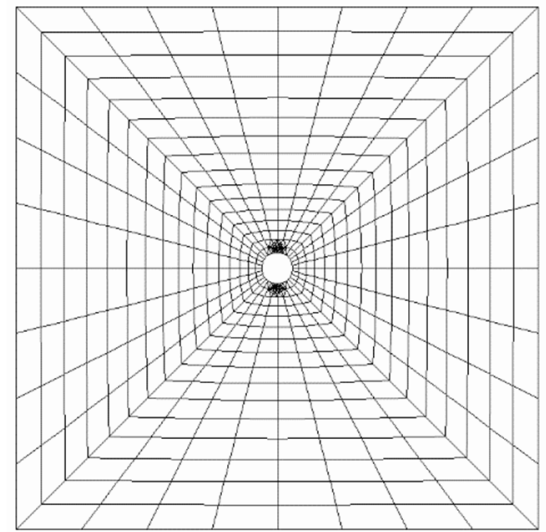

(a) $\mathrm{a}=0,69 \mathrm{~mm}$

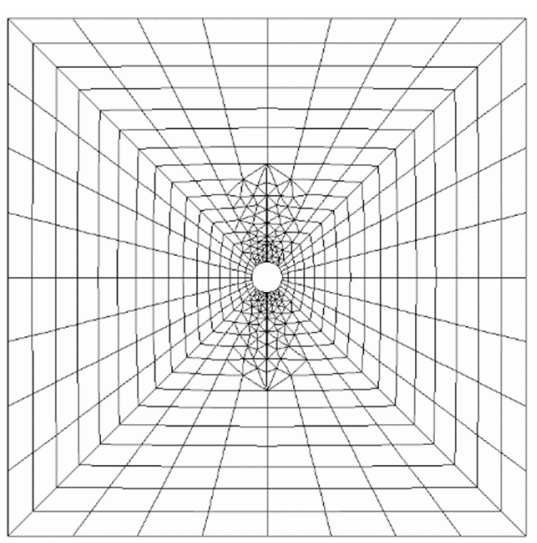

(b) $\mathrm{a}=16,19 \mathrm{~mm}$

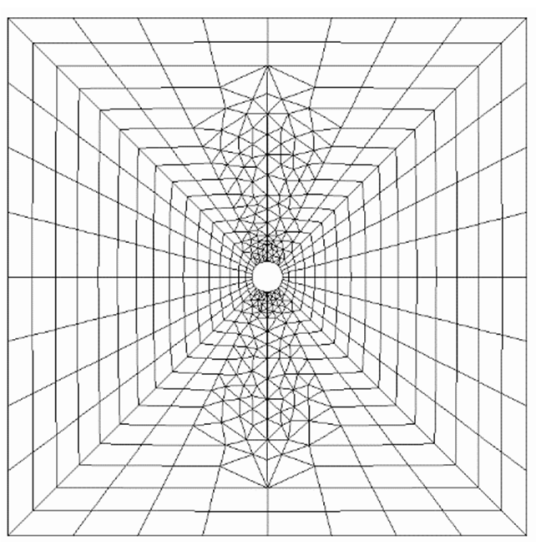

(c) $\mathrm{a}=36,19 \mathrm{~mm}$

Figure 5 - Simulação da propagação da fratura no programa FRANC2D. 


\section{R E F E R ÊNCIAS}

Table 1 - Parâmetros físicos do mármore Tennesse (HAIMSON, 1968; HAIMSON \& FAIRHURST, 1970).

\section{Parâmetros}

\begin{tabular}{|c|c|}
\hline Propriedade & Valor \\
\hline Módulo de elasticidade & $10,4 \times 10^{6} \mathrm{psi}(71,71 \mathrm{GPa})$ \\
\hline Coeficiente de Poisson & 0,28 \\
\hline Porosidade & 2,3 \\
\hline Resistência a compressão simples & 18000 psi $(124,106 \mathrm{MPa})$ \\
\hline $\begin{array}{l}\begin{array}{l}\text { Resistência a tração (ensaio } \\
\text { brasileiro) }\end{array} \\
\end{array}$ & 1800 psi $(12,411 \mathrm{MPa})$ \\
\hline
\end{tabular}

Table 2 - Tenacidade à fratura modo I, estimada pelas correlações de Backers (2004).

\begin{tabular}{cc}
\multicolumn{1}{c|}{ Parâmetros } \\
Correlação & Valor $\left(\mathbf{M P a} \cdot \mathbf{m}^{\mathbf{0 , 5}}\right)$ \\
\hline$K_{\mathrm{IC}}=0,05 \cdot E(\mathrm{GPa})$ & 3,59 \\
$K_{\mathrm{IC}}=0,02 \cdot \sigma_{\mathrm{c}}(\mathrm{MPa})$ & 2,48 \\
$K_{\mathrm{IC}}=0,25 \cdot \sigma_{\mathrm{t}}(\mathrm{MPa})$ & 3,10 \\
\hline
\end{tabular}

Table 3 - Fatores de intensidade de tensão modo I obtidos nas simulações no FRANC2D e na solução de referência de

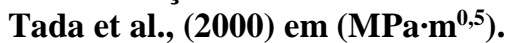

\begin{tabular}{|ccccccc}
\hline \multicolumn{7}{c|}{ Fatores de intensidade de tensão modo I } \\
\cline { 2 - 6 } \\
\cline { 2 - 6 } a \\
$(\mathrm{mm})$ & LC1 & LC2 & LC3 & $\begin{array}{c}\text { LC1+LC2+ } \\
\text { LC3 }\end{array}$ & $\begin{array}{c}\text { LC2+ } \\
\text { LC3 }\end{array}$ & $\begin{array}{c}\text { Tada } \\
\text { et al. } \\
(2000)\end{array}$ \\
\hline 0.69 & $-0,67$ & 1,23 & 1,53 & 2,08 & 2,76 & 2,68 \\
4.19 & $-1,41$ & 1,33 & 3,61 & 3,53 & 4,94 & 4,64 \\
6.19 & $-1,59$ & 1,26 & 4,26 & 3,93 & 5,52 & 5,27 \\
16.19 & $-2,44$ & 1,01 & 7,37 & 5,94 & 8,38 & 7,61 \\
26.19 & $-3,26$ & 0,97 & 10,2 & 7,93 & 11,2 & 9,37 \\
36.19 & $-4,26$ & 1,05 & 13,6 & 10,3 & 14,6 & 10,8 \\
\hline
\end{tabular}

\section{CONCLUSÃO}

Para o ensaio de laboratório de fraturamento hidráulico em um mármore estudado os modelos analíticos e de elementos finitos explicam o início da fratura.

Os modelos da solução de referência e de elementos finitos explicam a propagação da fratura. A manutenção do carregamento no interior da fratura é importante no processo de propagação. A inclusão de elementos finitos de interface na análise pode fortalecer a consistência dos resultados.

A obtenção de parâmetros de resistência das rochas por interpretações pela mecânica do contínuo é mais usual que nas abordagens pela mecânica da fratura. Como as duas abordagens explicam processos diferentes, se faz necessário melhorar o conhecimento da tenacidade à fratura das rochas.
BACKERS, T. Fracture toughness determination and micromechanics of rock under mode I and mode II loading. 2004. $94 \mathrm{f}$. Thesis (PhD in Geology) - University of Potsdam, Germany, 2004.

BRADLEY, W. B. Failure of inclined boreholes. Journal of Energy Resources Technology, v. 101, n. 4, p. 232-239, 1979.

CIMNE. GiD: The personal pre and post processor. 2017. Available at: <www.gidhome.com>. Accessed on November $29^{\text {th }}, 2017$.

FJÆR, E.; HOLT, R.M.; HORSRUD, P.; RAAEN, A.M. \& RISNES, R. Petroleum Related Rock Mechanics. $2^{\text {nd }}$ ed., Amsterdam: Elsevier, 2008.

HAIMSON, B.; FAIRHURST, C. Ln-situ stress determination at great depth by means of hydraulic fracturing. In: $11^{\text {th }}$ US SYMPOSIUM ON ROCK MECHANICS, 16-19 June 1969, Berkeley, California. Proceedings... New York: AIME, 1970, p. 559-584.

HAIMSON, B. Hydraulic fracturing in porous and nonporous rock and its potential for determining in-situ stresses at great depth. 1968. 234 f. Thesis (PhD in Geology), University of Minnesota, United States of America, 1968.

LAZARUS AND FREE PASCAL TEAM. Lazarus. 2014. Available at: <http://www.lazarus.freepascal.org/>. Accessed on March $13^{\text {th }}, 2014$.

SAMMIS, C. G.; ASHBY, M. F. The failure of brittle porous solids under compressive stress states. Acta metallurgica, v. 34, n. 3, p. 511-526, 1986.

TADA, H.; PARIS, P.C.; IRWIN, G.R. The stress analysis of cracks handbook. $3^{\text {rd }}$ ed. New York: ASME Press, 2000.

WAWRZYNEK, P.; INGRAFFEA, A. FRANC2D: A two dimensional crack propagation simulator. 1993. User's Guide. Version 3.1. Available at: <http://www.cfg.cornell.edu/doc/F2D_V3.1.pdf $>$. Accessed on February $3^{\text {rd }}, 2014$.

ZHAO, H.; LI, Z.; ZHU, C.; RU, Z. Reliability analysis models for hydraulic fracturing. Journal of Petroleum Science and Engineering, v. 162, p. 150-157, 2018.

NARDELLI, A. M. B.; GRIFFITH, J. J. Theoretical model for understanding corporate environmentalism in the Brazilian forestry sector. Revista Árvore, v. 27, n. 6, p. 855-869, 2003. 